\title{
METRIC INVARIANCE OF HAAR MEASURE
}

\author{
CHRISTOPH BANDT
}

\begin{abstract}
Let $d$ be a left invariant metric for a locally compact group $G$. We prove that isometric subsets of $(G, d)$ have equal Haar measure.
\end{abstract}

1. Introduction. In elementary geometry we learn that "congruent figures in plane have equal area". This concerns the Euclidean metric and it is not quite clear whether isometric sets with respect to other metrics on $R^{2}$ or $R^{n}$ have equal Lebesgue measure. Fickett and Mycielski [3] gave an affirmative answer for a large class of metrics including all norms. This result is extended below to all translation-invariant metrics on $R^{n}$.

In fact we consider a more general situation. Let $(X, d)$ be a metric space and $\mu$ a Borel measure on $X$. We say that $\mu$ is invariant with respect to $d$, or $d$-invariant, if isometric Borel sets of $X$ have equal measure. $D$ and $F$ are isometric if there is a mapping $f$ from $D$ onto $F$ with $d(f(x), f(y))=d(x, y)$ for all $x, y$ in $D$.

Ulam asked whether the Lebesgue product measure on Hilbert's cube is invariant with respect to certain familiar metrics. Although a number of positive results were obtained by Mycielski $[8,9]$ the question remains unresolved. We do not touch upon this problem here but confine our attention to Haar measure on locally compact groups $[6,10,4]$ for which the question of metric invariance can be answered in a simple way.

THEOREM 1. Let $(G, d)$ be a metric, locally compact group, and let $d$ be left invariant. Then any left Haar measure on $G$ is d-invariant.

Throughout the paper we shall assume that $d$ is a left invariant metric for the locally compact group $G, A$ is a compact subset of $G$ with nonempty interior and $\lambda$ is a Haar measure. For the proof of Theorem 1 it suffices to construct a $d$-invariant Borel measure $\mu$ on $G$ with $0<\mu(A)<\infty$. Since left translations are isometries, $\mu$ will be a Haar measure and hence every Haar measure on $G$ will be $d$-invariant.

To find $\mu$ we combine the Weil-Cartan construction of Haar measure with Hausdorff's measure construction.

Received by the editors February 8, 1982.

1980 Mathematics Subject Classification. Primary 28C10, 43A05; Secondary 28A12.

Key words and phrases. Haar measure, isometric sets, Hausdorff measure, fractional covering, multiple covering. 
2. Hausdorff measure. The $h$-dimensional Hausdorff measure $\mu^{h}$ on a metric space $(X, d)$ is defined by

$$
\mu^{h}(D)=\lim _{t \rightarrow 0} \inf \left\{\sum_{j=1}^{\infty} h\left(\delta\left(B_{j}\right)\right) \mid D \subseteq \bigcup_{j=1}^{\infty} B_{j}, \delta\left(B_{j}\right) \leqslant t\right\},
$$

where $D$ is a Borel set, $\delta(B)=\sup \{d(x, y) \mid x, y \in B\}$ denotes the diameter of the set $B$, and $h:\left[0, \infty\left[\rightarrow\left[0, \infty\left[\right.\right.\right.\right.$ is a Hausdorff function (nondecreasing, $\lim _{t \rightarrow 0} h(t)=$ $h(0)=0)$.

$\mu^{h}$ is a Borel measure ([5], cf. [11, 2]). Since we can require $B_{j} \subseteq D$ and $\delta\left(f\left(B_{j}\right)\right)=$ $\delta\left(B_{i}\right)$ then holds for any isometry $f: D \rightarrow F, \mu^{h}$ is $d$-invariant.

The problem consists in choosing the function $h$ in such a manner that $0<\mu(A)$ $<\infty$. In our case $X=G$, Kahnert [7] and Mycielski [8, 9] have suggested to take the functions $h_{1}(t)=1 / E(A, t)$ with $E(A, t)=\min \left\{k \mid A \subseteq \cup_{j=1}^{k} B_{j}, \delta\left(B_{j}\right) \leqslant t\right\}$ and $h_{2}(t)=\sup \{\lambda(B) \mid \delta(B) \leqslant t\}$.

It is easy to show that $\mu^{h_{1}}(A) \leqslant 1$ and $\mu^{h_{2}}(A) \geqslant \lambda(A)$ [7], but $\mu^{h_{1}}(A)=0$ and $\mu^{h_{2}}(A)=\infty$ are possible [1, Examples 1 and 2], probably even at the same time, so that $\mu^{h}$ will hardly prove Theorem 1 .

3. Fractional coverings. As a counterpart we examine the existence proofs for Haar measure. Haar's original method, as presented by Halmos [4, §58], is quite suitable for our purpose. If we replace $\lambda_{U}(C)=C: U / A: U$ by $\lambda_{t}(C)=E(C, t) / E(A, t)$ with $t>0$ and $t \rightarrow 0$, Halmos's procedure will show immediately that $\lambda$ is invariant with respect to isometries of $G$ onto itself, and that open isometric subsets of $G$ have equal measure (note that $\lambda_{t}$ is $d$-invariant, and use [4, Theorems 53.F and 53.C]). However, the nonconstructive character of the proof makes it difficult to improve on these assertions.

Cartan, following Weil, constructed Haar measure as a linear form on a function space, using coverings $f \leqslant \Sigma c_{j} \cdot g_{j}$ of a positive function $f$ by given functions $g_{j}$ with coefficients $c_{j} \geqslant 0$ instead of coverings $D \subseteq \cup B_{j}$ of a set $D$ (cf. [6, §15] or [10, Chapter II, §8]). This proof can hardly be connected with metrics, but the new coverings do apply successfully to Hausdorff measure.

Let $1_{D}, 1_{B}$ denote the characteristic functions of the sets $D, B_{j}\left(1_{D}(x)=1\right.$ for $x$ in $D, 0$ otherwise). An expression of the form $1_{D} \leqslant \Sigma c_{j} \cdot 1_{B}$, with $c_{j} \geqslant 0$ is called a fractional covering of $D$ by the sets $B_{j}$. Accordingly, the measure $\nu^{h}$ on a metric space $(X, d)$ defined by

$$
\nu^{h}(D)=\lim _{t \rightarrow 0} \inf \left\{\sum_{j=1}^{\infty} c_{j} \cdot h\left(\delta\left(B_{j}\right)\right) \mid 1_{D} \leqslant \sum_{j=1}^{\infty} c_{j} \cdot 1_{B_{i}}, c_{j} \geqslant 0, \delta\left(B_{j}\right) \leqslant t\right\}
$$

will be referred to as fractional Hausdorff measure.

As far as we know, this measure has been used first by Wegmann [14, Satz 3 and 4] as a tool for proving a theorem on Hausdorff dimension of products. In contemporary combinatorics, however, fractional coverings have already become popular: the relevant survey of Schrijver [13] comprises 175 references.

It is obvious that $\nu^{h} \leqslant \mu^{h}$. The fact that $\nu^{h}$ is a Borel measure can be verified as usual $[14,5,11,2]: \nu^{h}$ is $\sigma$-subadditive and additive for sets with positive distance. As in $\S 2$, metric invariance of $\nu^{h}$ is easy to see. 
4. Two Hausdorff functions. We return to our group $X=G$ and look for functions $h$ with $0<\nu^{h}(A)<\infty$. We shall see that $h_{2}(t)=\sup \{\lambda(B) \mid \delta(B) \leqslant t\}$ can be used as above, but $h_{1}$ has to be adapted to fractional coverings: Let $h_{3}(t)=1 / E^{\prime}(A, t)$ with

$$
E^{\prime}(A, t)=\inf \left\{\sum_{j=1}^{n} c_{j} \mid n \in N, 1_{A} \leqslant \sum_{j=1}^{n} c_{j} \cdot 1_{B_{i}}, c_{j} \geqslant 0, \delta\left(B_{j}\right) \leqslant t\right\}
$$

Proposition. (a) $\nu^{h_{2}}(D) \geqslant \lambda(D)$ for all Borel sets $D$ in $G$.

(b) If $\lim _{t \rightarrow 0} h_{2}(t) / h_{3}(t)<\infty$, then $0<\nu^{h_{2}}(A)<\infty$.

To prove (a) we apply the Haar integral $[6,10] .1_{D} \leqslant \sum_{j=1}^{\infty} c_{j} \cdot 1_{B_{1}}$ implies

$$
\lambda(D)=\int 1_{D} d \lambda \leqslant \int \sum c_{j} \cdot 1_{B_{1}} d \lambda=\sum c_{j} \cdot \lambda\left(B_{j}\right) \leqslant \sum c_{j} \cdot h_{2}\left(\delta\left(B_{j}\right)\right) .
$$

Thus all sums in the definition of $\nu^{h_{2}}(D)$ are $\geqslant \lambda(D)$, and so $\nu^{h_{2}}(D) \geqslant \lambda(D)$. In particular, $\nu^{h_{2}}(A) \geqslant \lambda(A)>0$. For (b) it remains to show $\nu^{h_{2}}(A)<\infty$. We have a constant $c$ and a sequence $\left(t_{k}\right)_{k \in N}, t_{k}>0, t_{k} \rightarrow 0$, with $h_{2}\left(t_{k}\right) / h_{3}\left(t_{k}\right)<c$ for all $k$. In other words, $E^{\prime}\left(A, t_{k}\right)<c / h_{2}\left(t_{k}\right)$. Thus there is a fractional covering $1_{A} \leqslant$ $\sum_{j=1}^{n_{k}} c_{j}^{k} \cdot 1_{B_{j}^{k}}$ with $c_{j}^{k} \geqslant 0, \delta\left(B_{j}^{k}\right) \leqslant t_{k}$ and $\sum c_{j}^{k} \leqslant c / h_{2}\left(t_{k}\right)$. Using this covering in the definition of $\nu^{h_{2}}(A)$ for $t=t_{k}$ we get $\inf \left\{\Sigma \cdots \leqslant t_{k}\right\} \leqslant \Sigma c_{j}^{k} \cdot h_{2}\left(t_{k}\right) \leqslant c$. Since this holds for all $k$, the limit $\nu^{h_{2}}(A)$ is also $\leqslant c$.

5. Cartan's approximation theorem for sets. The following statement says that "there are very economical fractional coverings by translations of any given open set". For simple coverings no similar assertion is possible, not even in Euclidean $R^{n}$ [12, Chapter 8].

THEOREM 2. Let $(G, d)$ be a locally compact metric group, where $d$ is left invariant, $A$ a compact set in $G, \lambda$ a left Haar measure and $\varepsilon>0$. Then there is a number $t_{0}>0$ with the following property. For any open set $B$ in $G$ with $\delta(B) \leqslant t_{0}$ there exist points $s_{1}, \ldots, s_{n}$ in $G$ and positive numbers $d_{1}, \ldots, d_{n}$ such that $\sum d_{i} \cdot 1_{s_{1} \cdot B} \geqslant 1_{A}$ and $\sum d_{i} \cdot \lambda\left(s_{i} \cdot B\right) \leqslant(1+\varepsilon) \cdot \lambda(A){ }^{1}$

(In all sums of this section, $i$ runs from 1 to $n$.) With this theorem we shall complete the proof of Theorem 1. For a given $\varepsilon>0$, choose $t \leqslant \min \left\{t_{0}(\varepsilon), \varepsilon\right\}$ such that $h_{2}$ is continuous in $t$. We find an open set $B$ with $\delta(B) \leqslant t$ and $h_{2}(t) \leqslant(1+\varepsilon)^{2}$ $\cdot \lambda(B)$. (Let $t^{\prime} \leqslant t$ with $h_{2}(t) \leqslant(1+\varepsilon) h_{2}\left(t^{\prime}\right)$, let $C$ be a set with $\delta(C) \leqslant t^{\prime}$ and $h_{2}\left(t^{\prime}\right) \leqslant(1+\varepsilon) \cdot \lambda(C)$, and let $B=\left\{x \mid d(x, C)<t-t^{\prime}\right\}$.) By Theorem 2 we have a covering $1_{A} \leqslant \Sigma d_{i} \cdot l_{s, B}$ with $\lambda(B) \cdot \sum d_{i} \leqslant(1+\varepsilon) \cdot \lambda(A)$. Consequently,

$$
\begin{aligned}
h_{2}(t) / h_{3}(t) & =h_{2}(t) \cdot E^{\prime}(A, t) \leqslant h_{2}(t) \cdot \sum d_{i} \\
& \leqslant(1+\varepsilon)^{2} \lambda(B) \cdot \sum d_{i} \leqslant(1+\varepsilon)^{3} \lambda(A) .
\end{aligned}
$$

Since $\varepsilon$ is arbitrary, this implies $\lim _{t \rightarrow 0} h_{2}(t) / h_{3}(t) \leqslant \lambda(A)$. Our proposition applies, $\mu=\nu^{h_{2}}$ is a $d$-invariant measure with $0<\mu(A)<\infty$, and Theorem 1 is proved.

${ }^{1}$ In the case $\lambda(A)=0, \Sigma d_{i} \lambda\left(s_{i} B\right) \leqslant \varepsilon$. 
It remains to prove Theorem 2 which is in fact only a modification of the following result of Cartan (cf. [6, p. 189] or [10, p. 115]), the key to a constructive proof of existence of Haar measure.

CARTAN'S THEOREM. Let $G$ be a locally compact group, $f$ a positive continuous function on $G$ with compact support and $\alpha>0$. Then there is a neighbourhood $U_{0}$ of the unit element $e$ in $G$ with the following property.

For any positive continuous function $g$ with support in $U_{0}$ there exist points $s_{1}, \ldots, s_{n}$ in the support of $f$ and positive numbers $c_{1}, \ldots, c_{n}$ such that

$$
\left|f(x)-\sum c_{i} \cdot g\left(s_{i}^{-1} \cdot x\right)\right| \leqslant \alpha \text { for all } x \text { in } G .
$$

To derive Theorem 2 from this result, let $c=\sqrt[3]{1+\varepsilon}$. Since $\lambda$ is regular and $A$ is compact, we find a neighbourhood $V=\{x \mid d(x, A)<b\}$ of $A$ such that $\bar{V}$ is compact and $\lambda(V) \leqslant c \cdot \lambda(A)$. Let $W=\{x \mid d(x, A)<b / 2\}$, and let $f: G \rightarrow[0,1]$ be a continuous function with $f(A)=\{1\}$ and $f(G-W)=\{0\}$. In other words, $1_{A} \leqslant f \leqslant 1_{W}$. Choose $\alpha>0$ such that $\alpha(1+c)<c-1$ and hence $(1+\alpha) /(1-\alpha)$ $<c$. Applying Cartan's theorem to $f$ and $\alpha$ we obtain $U_{0}$. Let us define $t_{0}=$ $\min \left\{b / 2, d\left(e, G-U_{0}\right)\right\}$, and let us prove that $t_{0}$ has the desired property.

Let $B$ be open and $\delta(B) \leqslant t_{0}$. Since $x^{-1} \cdot B \subseteq U_{0}$ for every $x$ in $B$, we shall just assume that $e \in B \subseteq U_{0}$. By regularity of $\lambda$, there is a closed set $C \subseteq B$ with $c \cdot \lambda(C) \geqslant \lambda(B)$. Let $g: G \rightarrow[0,1]$ be a continuous function with $1_{C} \leqslant g \leqslant 1_{B}$.

Since the support of $g$ is in $U_{0}$, Cartan's theorem says that there are points $s_{i}$ in $W$ and numbers $c_{1} \geqslant 0$ with

$$
1_{A}(x)-\alpha \leqslant f(x)-\alpha \leqslant \sum c_{i} \cdot g\left(s_{i}^{-1} \cdot x\right) \leqslant f(x)+\alpha \text { for } x \text { in } G .
$$

We write $g_{i}(x)=g\left(s_{i}^{-1} \cdot x\right)$ and $d_{i}=c_{i} /(1-\alpha)$. The definition of $g$ and $g_{i}$ implies $g_{1} \leqslant 1_{s_{i} B}$ and our first assertion

$$
1_{A} \leqslant \sum d_{i} \cdot g_{i} \leqslant \sum d_{i} \cdot 1_{s, B} \text {. }
$$

The function on the right-hand side is zero on $G-V$ since the $s_{i}$ are in $W$ and $d(W, G-V)>b / 2 \geqslant \delta(B)$. Thus (*) implies $\sum d_{i} \cdot g_{i} \leqslant c \cdot 1_{V}$. By the properties of Haar integral,

$$
\sum d_{i} \cdot \int g d \lambda=\sum d_{i} \cdot \int g_{i} d \lambda=\int \sum d_{i} \cdot g_{i} d \lambda \leqslant \int c \cdot 1_{V} d \lambda=c \cdot \lambda(V) .
$$

Using the definitions of $C, g, V$ and $c$ we finish the proof.

$$
\begin{aligned}
\sum d_{i} \cdot \lambda\left(s_{i} B\right) & =\lambda(B) \cdot \sum d_{i} \leqslant c \cdot \lambda(C) \cdot \sum d_{i} \leqslant c \cdot \int g d \lambda \cdot \sum d_{i} \\
& \leqslant c^{2} \cdot \lambda(V) \leqslant c^{3} \cdot \lambda(A)=(1+\varepsilon) \cdot \lambda(A) .
\end{aligned}
$$

\section{REFERENCES}

1. C. Bandt, Many measures are Hausdorff measures, Bull. Acad. Polon. Sci. Sèr. Sci. Math. (to appear).

2. H. Federer, Geometric measure theory, Springer-Verlag, Berlin and New York, 1969.

3. J. Fickett and J. Mycielski, A problem of invariance for Lebesgue measure, Colloq. Math. 42 (1979), $123-125$. 
4. P. R. Halmos, Measure theory, Van Nostrand, Princeton, N. J., 1950.

5. F. Hausdorff, Dimension und äusseres Mass, Math. Ann. 79 (1919), 157-179.

6. E. Hewitt and K. A. Ross, Abstract harmonic analysis, Vol. 1, Springer-Verlag, Berlin and New York, 1963

7. D. Kahnert, Haar-Mass und Hausdorff-Mass, Measure Theory (Proc. Conf. Oberwolfach, 1975), Lecture Notes in Math., vol. 541, Springer-Verlag, Berlin and New York, 1976, pp. 13-23.

8. J. Mycielski, Remarks on invariant measures in metric spaces, Colloq. Math. 32 (1974), 109-116.

9. , A conjecture of Ulam on the invariance of measures in Hilbert's cube, Studia Math. 60 (1977), 1-10.

10. L. Nachbin. The Haar integral, Van Nostrand, Princeton, N. J., 1965.

11. C. A. Rogers, Hausdorff measures, Cambridge Univ. Press, London and New York, 1970.

12. __ Packing and covering, Cambridge Univ. Press, London and New York, 1964.

13. A. Schrijver, Fractional packing and covering, Packing and Covering in Combinatorics, Math. Centre Tracts, vol. 106, Amsterdam, 1979, pp. 201-274.

14. H. Wegmann, Die Hausdorff-Dimension von kartesischen Produkten metrischer Räume, J. Reine Angew. Mat. 246 (1971), 46-75.

Department of Mathematics, AdDis Ababa University, POB 1176, AdDis ABABA, Ethiopia 